\title{
Prioritization of natural extracts by LC-MS-PCA for the identification of new photosensitizers for photodynamic therapy
}

\begin{abstract}
Photodynamic therapy (PDT) is an alternative treatment for cancer that involves administration of a photosensitive drug or photosensitizer that localizes at the tumor tissue followed by in situ excitation at an appropriate wavelength of light. Tumour tissues are then killed by cytotoxic reactive oxygen species generated by the photosensitizer. Targeted excitation and photokilling of affected tissues is achieved through focal light irradiation, thereby minimizing systemic side effects to the normal healthy tissues. Currently, there are only a small number of photosensitizers that are in the clinic and many of these share the same structural core based on cyclic tetrapyrroles. This paper describes how metabolic tools are utilized to prioritize natural extracts to search for structurally new photosensitizers from Malaysian biodiversity. As proof of concept, we analyzed 278 photocytotoxic extracts using a hyphenated technique of liquid chromatography-mass spectrometry coupled with principal component analysis (LC-MS-PCA) and prioritized 27 extracts that potentially contained new photosensitizers for chemical dereplication using an in-house UPLC-PDA-MSPhotocytotoxic assay platform. This led to the identification of 2 new photosensitizers with cyclic tetrapyrrolic structures, thereby demonstrating the feasibility of the metabolic approach.
\end{abstract}

Keyword: Natural extracts; Tumour tissues; Photosensitizers; Photodynamic therapy 